\title{
On Minimal Moment of Inertia Polyominoes`
}

\author{
Srečko Brlek, Gilbert Labelle, and Annie Lacasse \\ Laboratoire de Combinatoire et d'Informatique Mathématique, \\ Université du Québec à Montréal, \\ CP 8888, Succ. Centre-ville, Montréal (QC) Canada H3C3P8 \\ \{brlek.srecko, labelle.gilbert\}@uqam.ca, annie.lacasse@gmail.com
}

\begin{abstract}
We analyze the moment of inertia $\mathrm{I}(S)$, relative to the center of gravity, of finite plane lattice sets $S$. We classify these sets according to their roundness: a set $S$ is rounder than a set $T$ if $\mathrm{I}(S)<\mathrm{I}(T)$. We show that roundest sets of a given size are strongly convex in the discrete sense. Moreover, we introduce the notion of quasi-discs and show that roundest sets are quasi-discs. We use weakly unimodal partitions and an inequality for the radius to make a table of roundest discrete sets up to size 40 . Surprisingly, it turns out that the radius of the smallest disc containing a roundest discrete set $S$ is not necessarily the radius of $S$ as a quasi-disc.
\end{abstract}

Keywords: Discrete sets, moment of inertia, polyominoes, lattice paths.

\section{Introduction}

In this paper we consider plane sets up to translations. By a discrete set we mean a finite set of lattice points or a finite union of lattice closed unit squares (pixels) (Figure1(a)).

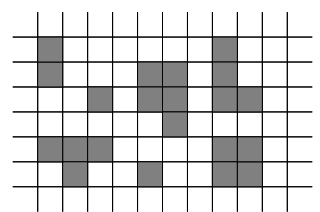

(a)

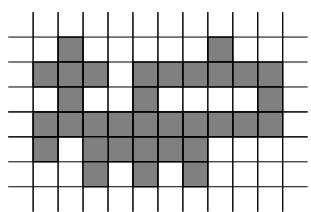

(b)

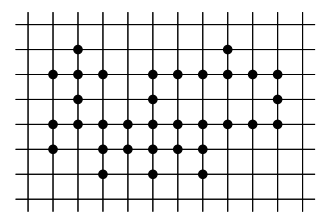

(c)

Fig. 1. (a) Discrete set (b) a typical polyomino and (c) its corresponding animal

In particular, the word polyomino means a finite union of pixels in the plane whose boundary consists of a disjoint union of simple closed polygonal paths using 4-connectedness (Figure [1 (b)). These sets are well-known combinatorial objects in discrete geometry. The dual of a polyomino, usually called animal,

\footnotetext{
^ With the support of NSERC (Canada).
} 
consists of the set of centers of its pixels. Using a $\left(\frac{1}{2}, \frac{1}{2}\right)$ shift, we may always assume that an animal is a subset of the discrete plane $\mathbb{Z} \times \mathbb{Z}$. Moreover, a polyomino is called $v$-convex (resp. $h$-convex) if all its columns (resp. rows) are connected (Figure 2(a),(b)). A polyomino is hv-convex (Figure 2(c)) if all its columns and rows are connected and strongly-convex (Figure 2(d)) if given any two points $u$ and $v$ in its corresponding animal, the lattice points $w$ in the segment $[u, v]$ are all in the animal. This notion coincides with the MP-convexity of Minsky and Papert [1] since animals are connected.

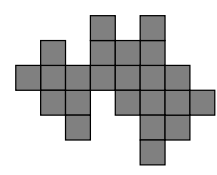

(a)

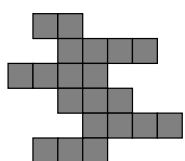

(b)

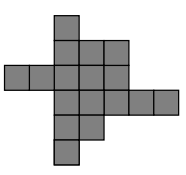

(c)

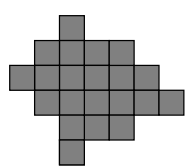

(d)

Fig. 2. A polyomino (a) v-convex (b) h-convex (c) hv-convex (d) strongly-convex

Our goal is to study the roundest discrete sets $S$ of $N$ pixels (or $N$ points) having minimal moment of inertia $\mathrm{I}(S)$ relatively to the center of gravity, a problem raised in a previous paper [2 in the context of designing incremental algorithms based on discrete Green theorem. This notion of roundness is distinct from the one given in [3] where they consider minimizing the site perimeter of lattice sets, that is the number of points with Manhattan distance 1 from the sets. For a given $N$, minimizing $\mathrm{I}(S)$ is equivalent to minimizing $\mathrm{I}(A)$, where $A$ is the associated animal. To simplify notations and computations, the plane is identified with the complex plane $\mathbb{C}$ and $\mathbb{Z} \times \mathbb{Z}$ is identified with $\mathbb{Z}+i \mathbb{Z}$.

Section 2 recalls some basics about moment of inertia of discrete sets. Section 3 is devoted to properties of roundest discrete sets. More precisely, we establish a useful lemma concerning the moment of inertia of a union of discrete sets. We also introduce discrete quasi-discs and apply our lemma to establish that roundest discrete sets are strongly convex quasi-discs. Using the combinat package of the Maple formal tool [4] a method is developed for computing the roundest discrete sets according to size (Table 1) and some parameters associated to them (Table 2). Finally, we show how to extend our results to other kinds of lattices and to higher dimensions. Due to the lack of space, the proofs will appear in full detail in an extended version and also in the $\mathrm{PhD}$ dissertation of the third author [5].

\section{Continuous and Discrete Moments of Inertia}

We recall the definitions of the basic geometric parameters:

Definition 1. Let $S$ be a measurable subset of $\mathbb{C}$ such that

$$
\iint_{S}|z|^{2} d x d y<\infty .
$$


The center of gravity $g$ and the moment of inertia $\mathrm{I}(S)$, relative to the center of gravity are defined by the following equations:

$$
g=g(S)=\frac{1}{\operatorname{Area}(S)} \iint_{S} z d x d y
$$

and

$$
\mathrm{I}(S)=\iint_{S}|z-g|^{2} d x d y=\iint_{S}|z|^{2} d x d y-\frac{1}{\operatorname{Area}(S)}\left|\iint_{S} z d x d y\right|^{2},
$$

where $\operatorname{Area}(S)=\iint_{S} d x d y$.

In particular, if $S=P_{1} \cup P_{2} \cup \cdots \cup P_{N}$ is a union of $N$ distinct pixels, the condition $\iint_{P_{1} \cup P_{2} \cup \cdots \cup P_{N}}|z|^{2} d x d y<\infty$ is obviously satisfied and $g(S)$ and $\mathrm{I}(S)$ are well-defined. Moreover, for any single pixel $P$ we have $\mathrm{I}(P)=\frac{1}{6}$ and its center of gravity corresponds to its geometrical center.

Definition 2. Let $T=\left\{a_{1}, a_{2}, \cdots, a_{N}\right\} \subseteq \mathbb{C}$ be a set of $N$ distinct points in the complex plane where the point $a_{k}$ has a mass $m_{k}$ for $k=1, \cdots, N$. The center of gravity $g$ and the moment of inertia $\mathrm{I}(T)$, relative to the center of gravity are defined by

$$
g=g(T)=\frac{1}{m_{1}+\cdots+m_{N}} \sum_{k=1}^{N} m_{k} a_{k}
$$

and

$$
\begin{aligned}
\mathrm{I}(T)=\sum_{k=1}^{N} m_{k}\left|a_{k}-g\right|^{2} & =\sum_{k=1}^{N} m_{k}\left|a_{k}\right|^{2}-\frac{1}{m_{1}+\cdots+m_{N}}\left|\sum_{k=1}^{N} m_{k} a_{k}\right|^{2} \\
& =\frac{1}{m_{1}+\cdots+m_{N}} \sum_{k<l} m_{k} m_{l}\left|a_{k}-a_{l}\right|^{2} .
\end{aligned}
$$

\section{Properties of Roundest Discrete Sets}

Any measurable (or finite) subset $S \subseteq \mathbb{C}$ satisfying (1), can be represented by its center of gravity $g$ with mass $\iint_{S} d x d y$ (or mass $|S|$ ). More generally, any family $S_{1}, S_{2}, \cdots, S_{N}$ of measurable (or finite) subsets $S_{k} \subseteq \mathbb{C}$ can be represented by the family $\left(g_{1}, g_{2}, \cdots, g_{N}\right)$ of their corresponding centers of gravity $g_{k}$ having mass $m_{k}=\iint_{S_{k}} d x d y$ (or mass $m_{k}=\left|S_{k}\right|$ ). From now on, we assume that every measurable subset of $\mathbb{C}$, satisfies (1).

The following useful lemma is a consequence of the classical parallel axis theorem [6] stating that, for any point $p$ and any measurable (or finite) set $S$, the moment of inertia of $S$ relative to $p$, denoted $\mathrm{I}_{p}(S)$ and defined by

$$
\mathrm{I}_{p}(S)=\iint_{S}|z-p|^{2} d x d y \quad\left(\text { or } \sum_{k=1}^{N} m_{k}\left|a_{k}-p\right|^{2}\right),
$$


satisfies

$$
\mathrm{I}_{p}(S)=\mathrm{I}(S)+m|p-g|^{2},
$$

where $g=g(S)$ and $m$ is the mass of $S$.

Lemma 1. Let $S_{1}, S_{2}, \cdots, S_{N}$ be disjoint measurable subsets $\subseteq \mathbb{C}$. Then

$$
\begin{aligned}
\mathrm{I}\left(S_{1} \cup \cdots \cup S_{N}\right) & =\sum_{k=1}^{N} \mathrm{I}\left(S_{k}\right)+\mathrm{I}\left(\left\{g_{1}, \cdots, g_{N}\right\}\right) \\
& =\sum_{k=1}^{N} \mathrm{I}\left(S_{k}\right)+\sum_{k=1}^{N} m_{k}\left|g_{k}\right|^{2}-\frac{1}{m}\left|\sum_{k=1}^{N} m_{k} g_{k}\right|^{2} \\
& =\sum_{k=1}^{N} \mathrm{I}\left(S_{k}\right)+\frac{1}{m} \sum_{k<l} m_{k} m_{l}\left|g_{k}-g_{l}\right|^{2},
\end{aligned}
$$

where $g_{k}$ is the center of gravity of $S_{k}$ with mass $m_{k}=\iint_{S_{k}} d x d y$ (or mass $\left.m_{k}=\left|S_{k}\right|\right)$ and $m=m_{1}+\cdots+m_{N}$.

\subsection{Roundest Discrete Sets Are Strongly Convex}

In order to analyse convexity properties of sets of minimal moment of inertia we need the following lemma.

Lemma 2. Let $L$ be a fixed line in the complex plane $\mathbb{C}$ and $c \notin L$ an arbitrary point with mass $p$. Let $d$ be the point on $L$ such that $[c, d]$ is the perpendicular segment to $L$. Let $a$ and $b$ be two distinct points in $L$ having mass $m, n$ respectively. We consider the following two cases:

Case 1. If $a$ and $b$ are both of the same side of $d$ on $L$ and

$$
0 \leq|a-d|<|b-d|,
$$

then the moment of inertia, $\mathrm{I}(\{a, b, c\})$, strictly decreases as $b$ moves towards a along the line $L$ (Figure 3 (i)).

Case 2. If $a$ and $b$ are on different sides of $d$ on $L$, then $\mathrm{I}(\{a, b, c\})$ strictly decreases as a or $b$ moves towards $d$ along the line L (Figure 3 (ii)).

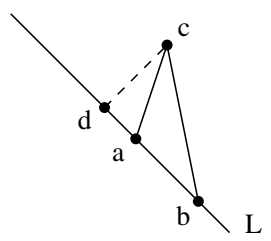

(i)

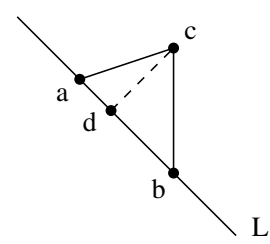

(ii)

Fig. 3. (i) Case 1 and (ii) Case 2 of Lemma 2 
Theorem 1. Let $N \geq 1$ and $S \subseteq \mathbb{C}$ be an arbitrary union of $N$ distinct lattice pixels (or $N$ lattice points) each having unit mass. If $S$ is of minimal moment of inertia, then $S$ is a strongly convex polyomino.

Proof. (Sketch) It is sufficient to consider the case where $S$ is a set of $N \geq 2$ lattice points. Then, by Definition 2, $\mathrm{I}(S)=q / N$, where $q \in \mathbb{N}^{*}$. Suppose that $\mathrm{I}(S)$ is minimal and that $S$ is not strongly convex. Then, $\exists u, v \in S$ such that the segment $[u, v]$ contains a point (hole) $w \in \mathbb{Z}+i \mathbb{Z}$ such that $w \notin S$. Let

$$
\begin{aligned}
& A=\{z \in S: \exists t \in \mathbb{Q}, z=u+t(v-u), t \leq 0\} \\
& B=\{z \in S: \exists t \in \mathbb{Q}, z=u+t(v-u), t \geq 1\}, \\
& C=S \backslash(A \cup B),
\end{aligned}
$$

where $a=g(A), b=g(B), c=g(C)$. Then by Lemmas 1 and 2, we can translate $A$ (or $B$ ) towards $w$ to obtain a set $A^{\prime}$ (or $B^{\prime}$ ) such that the set $S^{\prime}=A^{\prime} \cup B \cup$ $C \quad\left(\right.$ or $\left.S^{\prime}=A \cup B^{\prime} \cup C\right)$ has smaller moment of inertia $\mathrm{I}\left(S^{\prime}\right)<\mathrm{I}(S)$ and such that $\left|S^{\prime}\right|=|S|=N$ and $w \in S^{\prime}$. If the set $S^{\prime}$ still contains a hole we repeat the previous construction to sets $S^{\prime}, S^{(2)}, \cdots$ such that $\mathrm{I}(S)>\mathrm{I}\left(S^{\prime}\right)>\mathrm{I}\left(S^{(2)}\right)>$ $\mathrm{I}\left(S^{(3)}\right)>\cdots$. At each step, filling such a hole, decreases the moment of inertia by at least $\frac{1}{N}$. After a finite number of steps, this process must terminate and the resulting set $S^{(k)}$ must be a strongly convex set which is also an animal since it is, in particular, $h v$-convex.

\subsection{Roundest Discrete Sets Are Discrete Quasi-Discs}

Much more can be said. We now show that roundest polyominoes are nearly discs in the following sense:

Definition 3. Let $c \in \mathbb{C}, S \subseteq \mathbb{Z}+i \mathbb{Z}$ be a finite set of lattice points, and $r=\max _{s \in S}|s-c|$. Then $S$ is called a discrete

(i) disc centered at $c$ of radius $r$ if

$$
S=\{z:|z-c| \leq r\} \cap(\mathbb{Z}+i \mathbb{Z}),
$$

(ii) quasi-disc centered at $c$ of radius $r$ if

$$
\{z:|z-c|<r\} \cap(\mathbb{Z}+i \mathbb{Z}) \subseteq S \subseteq\{z:|z-c| \leq r\} \cap(\mathbb{Z}+i \mathbb{Z}) .
$$

A disc and a quasi-disc of radius $r=5$ are shown in Figure 4 (a) and (b) respectively. Note that every lattice point on the circumference must belong to a disc while at least only one is necessary in the case of quasi-disc. In both cases, every lattice point lying within the circumference must belong to the disc and quasi-disc.

Theorem 2. Let $S$ be a polyomino having $N$ pixels with minimal moment of inertia, that is a roundest polyomino. Let $A$ be its associated animal and $g=$ $g(A)$ be its center of gravity. Then $A$ is a quasi-disc centered at $g$ with radius $r=\max _{a \in A}|g-a|=\left|g-a_{0}\right|$, with $a_{0} \in A$. 


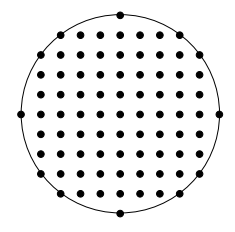

(i)

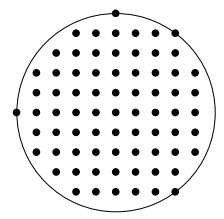

(ii)

Fig. 4. (i) A discrete disc (ii) a discrete quasi-disc

Figure 5 (a) illustrates Theorem 2 with $N=5$. By contraposition, the $7 \times 7$ lattice set $A$ is not minimal since the $\operatorname{disc} C_{a_{0}}=\left\{z \in \mathbb{C}:|z-g| \leq\left|g-a_{0}\right|\right\}$ contains lattice points not in $A$ (Figure 5 (b)). Note that the converse is false since, for $N=3$, the quasi-disc of Figure 5 (c) is not minimal (with $\mathrm{I}=2$ ). The minimal one for $N=3$ (with $\mathrm{I}=\frac{4}{3}<2$ ) is shown in Figure 5 (d).

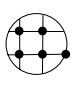

(a)

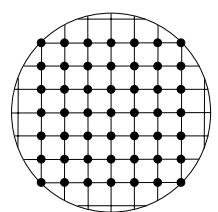

(b)

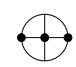

(c)

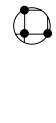

(d)

Fig. 5. Illustration of Theorem 2 and of the falsity of its converse

To pursue our study of roundest discrete sets we need a finer analysis. In particular, given $N$, the following result gives an upper bound for the radius $r$ of the $\operatorname{disc} C_{a_{0}}$.

Lemma 3. Let $A$ be a roundest animal having $N$ points. The radius $r=\mid a_{0}-$ $g\left|=\max _{a \in A}\right| a-g \mid$ of the disc $C_{a_{0}}$ centered at $g=g(A)$ satisfies

$$
r \leq \frac{1}{\sqrt{2}}+\sqrt{\frac{N}{\pi}} .
$$

\subsection{Generation of Roundest Discrete Sets According to Size}

In order to generate all the roundest animals of a given size $N$, we classify them according to their vertical projections. Let $A$ be a roundest animal of size $N$ with projections $\left(n_{1}, n_{2}, \cdots, n_{s}\right)$ with $N=n_{1}+n_{2}+\cdots+n_{s}$. Then, by convexity property, the sequence $n_{1}, n_{2}, \cdots, n_{s}$ must satisfy

$$
0<n_{1} \leq \cdots \leq n_{k}<n_{k+1}=\cdots=n_{l-1}>n_{l} \geq \cdots \geq n_{s-1} \geq n_{s}>0 .
$$

Such sequences are called weakly unimodal partitions of $N$ (or stack or planar partitions of $N$ ), see Stanley [7, Section 2.5, p. 76. Surprisingly, it turns out that 
any such sequence of projections corresponds to 0,1 or 2 roundest animal of size $N$. More precisely, we have the following result.

Lemma 4. Let $\left(n_{1}, n_{2}, \cdots, n_{s}\right)$ be a weakly unimodal sequence with $N=n_{1}+$ $\cdots+n_{s}$. Then among all animals having these vertical projections,

(i) there is a unique animal A, with minimal moment of inertia, if $n_{1}, n_{2}, \cdots, n_{s}$ have the same parity;

(ii) otherwise, there are exactly two animals $A, A^{\prime}$, having these projections, with minimal moment of inertia.

These strongly convex animals $A$ and $A^{\prime}$ are maximally symmetrically disposed along the $x$-axis (Figure 6). Moreover, the moment of inertia of $A$ (and $A^{\prime}$ ) is given by the formula

$$
\begin{aligned}
\mathrm{I}(A)=\frac{1}{12} \sum_{k=1}^{s} n_{k}^{3}-\frac{1}{12} N & +\sum_{k=1}^{s} k^{2} n_{k}-\frac{1}{N}\left(\sum_{k=1}^{s} k n_{k}\right)^{2} \\
& +\frac{1}{4 N}\left(\sum_{n_{k} \text { even }} n_{k}\right)\left(\sum_{n_{k} \text { odd }} n_{k}\right) .
\end{aligned}
$$

Using the computer algebra software Maple 4], we now generate all roundest animals with size $N \leq 40$. Our strategy is the following: We first encode the weakly unimodal sequences by

$$
(\lambda, b, h, \mu)
$$

where $\lambda, \mu$ are integer partitions and $b, h \in \mathbb{N}^{*}$. The sequences $\left(n_{1}, \cdots, n_{s}\right)$ are given by

$$
\lambda_{1} \leq \lambda_{2} \leq \cdots \leq \lambda_{k}<\underbrace{h=\cdots=h}_{b}>\mu_{l} \geq \mu_{l-1} \geq \cdots \geq \mu_{1},
$$

with $|\lambda|+b h+|\mu|=N$ (Figure 6).

Then, using the combinat package, we generate all $(\lambda, b, h, \mu)$ such that the associated animal $A$ minimizes the moment of inertia $\mathrm{I}(A)$ given by formula

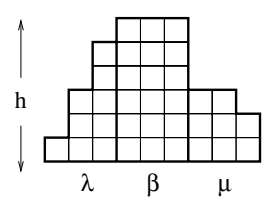

(a)

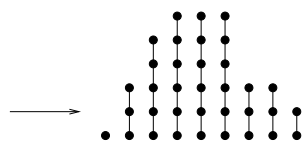

(b)

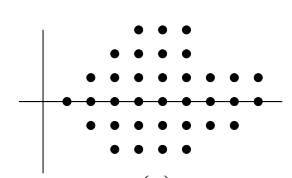

(c)

Fig. 6. (a) $(\lambda, b, \mu), \quad$ (b) $\left(n_{1}, \cdots, n_{s}\right), \quad$ (c) animal $S$ to test 
(3). Taking Lemma 3 into account, we restrict the generation of the 4-tuples $(\lambda, b, h, \mu)$ to those satisfying the further conditions

$$
s \leq 2 r+1 \text { and } h \leq 2 r+1
$$

that is

$$
\max (s, h) \leq\left\lfloor\sqrt{2}+2 \sqrt{\frac{N}{\pi}}+1\right\rfloor .
$$

In appendix, Table 1 gives, for each $N \leq 40$, a set of representatives, up to dihedral symmetry, of the roundest animals of size $N$. Various parameters associated to these roundest animals are given in Table 2. The first five columns give the size $N$, the vertical projections, the moment of inertia, the center of gravity and the radius of the circle $C_{a_{0}}$ of the roundest animals, up to dihedral symmetry.

Remark. Let $C_{\min }=\left\{z:|z-c| \leq r_{\min }\right\}$, be the smallest closed disc containing a given roundest animal $A$ of size $N$. The value of $c$ and $r_{\min }$ are given in the last two columns of Table 2. One may think that $A$ is a quasi-disc centered at $c$ having radius $r_{\min }$. In other words, we can replace the $\operatorname{disc} C_{a_{0}}$ of Theorem 2 by $C_{\text {min }}$. It turns out that this is false in general. As an example, let $A$ be the roundest animal of size $N=17$ having projections $[4,5,5,3]$ and $r_{\min }=\sqrt{5}$ be the radius of the smallest closed disc containing $A$ shown below.

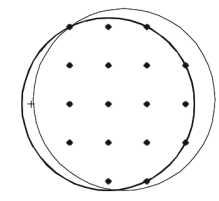

Then $A$ is not a quasi-disc of radius $r_{\min }=\sqrt{5}$ : indeed, the animal $A$ together with the discs $C_{a_{0}}$ and $C_{\text {min }}$ shows that the lattice point + (cross) belongs to the open $\operatorname{disc} C_{\min }^{\circ}$ but is not an element of $A$. This means that $A$ is not a quasi-disc corresponding to $C_{\min }$.

Conjecture. There exists an infinite family of roundest animals $A$ which are not a quasi-disc of radius $r_{\min }$, where $r_{\min }$ is the radius of the smallest closed disc containing $A$.

It turns out that the only occurence of such animal, up to $N=40$, is the animal above with $N=17$.

\section{Concluding Remarks}

While the recognition of digital straight lines is a well understood problem 8, both from the Euclidean and combinatorial approaches, the recognition of circles is still a challenging problem in discrete geometry. The border of discs or 
quasi-discs introduced in section 3.2, appears as a good candidate for a circle. This fact begs for a thorough study of their properties, perhaps useful for circle recognition.

The above results can be extended to other families of lattices. For instance, in the context of regular triangular lattices, a discrete set $S$ is a union of $N$ distinct closed equilateral triangles and the set $A$ of the centers of these triangles satisfies the following formula,

$$
\mathrm{I}(S)=\mathrm{I}(A)+N \cdot \mathrm{I}(T),
$$

where $T$ is the unit triangle of the lattice. The lattice set $\mathbb{Z}+i \mathbb{Z}$ must be replaced by the set $\mathbb{T} \subseteq \mathbb{C}$ of the centers of all lattice triangles. The associated notions of strong convexity, (discrete) disc and quasi-disc are easily defined using $\mathbb{T}$. Theorems 1 and 2 still hold. The constant $\frac{1}{\sqrt{2}}$ of Lemma 3 must be replaced by a suitable constant $\alpha$ (according to the lattice). The computation of the roundest discrete sets can be established using an adaptation of the strategy described in Section 3.4. Moreover, extensions to higher dimensional lattices are also possible.

Acknowledgement. The authors are grateful to the anonymous referees for the useful and accurate comments provided.

\section{References}

1. Minsky, M., Papert, S.: Perceptrons: An Introduction to Computational Geometry. MIT Press, Cambridge (1969)

2. Brlek, S., Labelle, G., Lacasse, A.: The discrete green theorem and some applications in discrete geometry. Theoret. Comput. Sci. 346(2), 200-225 (2005)

3. Altshuler, Y., Yanovsky, V., Vainsencher, D., Wagner, I.A., Bruckstein, A.M.: On minimal perimeter polyminoes. In: Kuba, A., Nyúl, L.G., Palágyi, K. (eds.) DGCI 2006. LNCS, vol. 4245, pp. 17-28. Springer, Heidelberg (2006)

4. Heck, A.: Introduction to Maple, 3rd edn. Springer, New York (2003)

5. Lacasse, A.: Contributions à l' analyse de figures discrètes en dimension quelconque. PhD thesis, Université du Québec à Montréal, Montréal (2008)

6. Feynman, R.P., Leighton, R.B., Sands, M.: The Feynman lectures on physics. Mainly mechanics, radiation, and heat, vol. 1. Addison-Wesley Publishing, Reading (1963)

7. Stanley, R.P.: Enumerative combinatorics. Cambridge Studies in Advanced Mathematics, vol. 1, vol. 49. Cambridge University Press, Cambridge (1997), with a foreword by Gian-Carlo Rota, Corrected reprint of the 1986 original

8. Klette, R., Rosenfeld, A.: Digital straightness-a review. Discrete Appl. Math. 139(1-3), 197-230 (2004) 


\section{Appendix}

Table 1. The roundest animals of size $N \leq 40$ (up to dihedral symmetry)

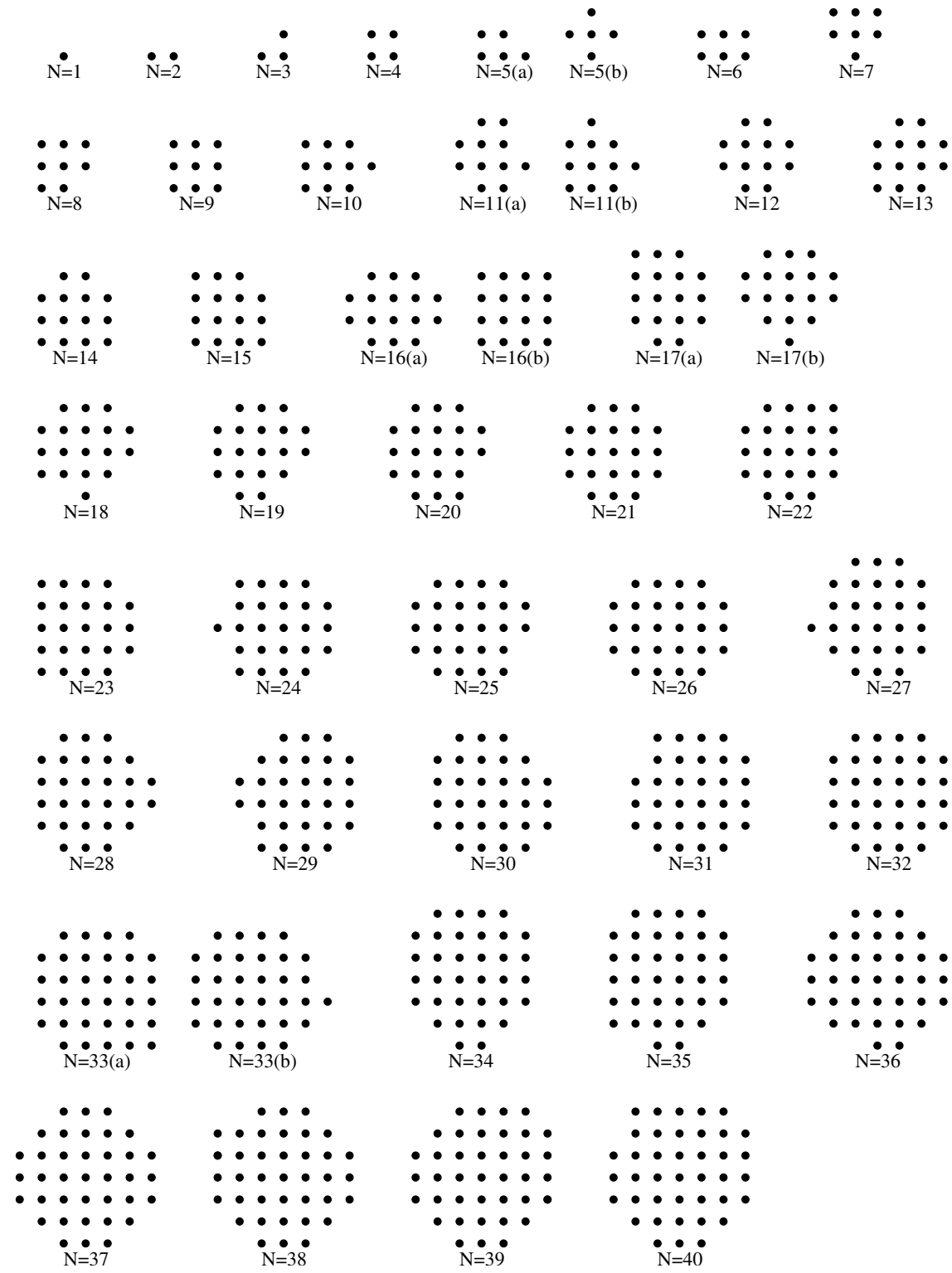


Table 2. Parameters associated to the roundest animals $(N \leq 40)$, up to symmetry

\begin{tabular}{|c|c|c|c|c|c|c|}
\hline$N$ & vertical projections & $\mathrm{I}(A)$ & $g(A)$ & $r=\max _{a \in A}|a-g|$ & $c(A)$ & $r_{\min }(A)$ \\
\hline 1 & [1] & 0 & $(1,0)$ & 0 & $(0,0)$ & 0 \\
\hline 2 & {$[1,1]$} & $\frac{1}{2}$ & $\left(\frac{3}{2}, 0\right)$ & $\frac{1}{2}$ & $\left(\frac{3}{2}, 0\right)$ & $\frac{1}{2}$ \\
\hline 3 & {$[1,2]$} & $\frac{4}{3}$ & $\left(\frac{5}{3}, \frac{1}{3}\right)$ & $\frac{1}{3} \sqrt{5}$ & $\left(\frac{3}{2}, \frac{1}{2}\right)$ & $\frac{1}{2} \sqrt{2}$ \\
\hline 4 & {$[2,2]$} & 2 & $\left(\frac{3}{2}, \frac{1}{2}\right)$ & $\frac{1}{2} \sqrt{2}$ & $\left(\frac{3}{2}, \frac{1}{2}\right)$ & $\frac{1}{2} \sqrt{2}$ \\
\hline $5 \mathrm{a}$ & {$[2,2,1]$} & 4 & $\left(\frac{9}{5}, \frac{2}{5}\right)$ & $\frac{2}{5} \sqrt{10}$ & $\left(2, \frac{1}{2}\right)$ & $\frac{1}{2} \sqrt{5}$ \\
\hline $5 \mathrm{~b}$ & {$[1,3,1]$} & 4 & $(2,0)$ & 1 & $(2,0)$ & 1 \\
\hline 6 & {$[2,2,2]$} & $\frac{33}{6}$ & $\left(2, \frac{1}{2}\right)$ & $\frac{1}{2} \sqrt{5}$ & $\left(2, \frac{1}{2}\right)$ & $\frac{1}{2} \sqrt{5}$ \\
\hline 7 & {$[2,3,2]$} & $\frac{52}{7}$ & $\left(2, \frac{2}{7}\right)$ & $\frac{9}{7}$ & $\left(2, \frac{1}{4}\right)$ & $\frac{5}{4}$ \\
\hline 8 & {$[3,3,2]$} & $\frac{78}{8}$ & $\left(\frac{15}{8}, \frac{1}{8}\right)$ & $\frac{1}{8} \sqrt{130}$ & $(2,0)$ & $\sqrt{2}$ \\
\hline 9 & {$[3,3,3]$} & $\frac{108}{9}$ & $(2,0)$ & $\sqrt{2}$ & $(2,0)$ & $\sqrt{2}$ \\
\hline 10 & {$[3,3,3,1]$} & $\frac{156}{10}$ & $\left(\frac{11}{5}, 0\right)$ & $\frac{9}{5}$ & $\left(\frac{7}{3}, 0\right)$ & $\frac{5}{3}$ \\
\hline $11 \mathrm{a}$ & {$[2,4,4,1]$} & $\frac{212}{11}$ & $\left(\frac{26}{11}, \frac{5}{11}\right)$ & $\frac{1}{11} \sqrt{349}$ & $\left(\frac{5}{2}, \frac{1}{2}\right)$ & $\frac{1}{2} \sqrt{10}$ \\
\hline $11 \mathrm{~b}$ & {$[3,4,3,1]$} & $\frac{212}{11}$ & $\left(\frac{24}{11}, \frac{2}{11}\right)$ & $\frac{2}{11} \sqrt{101}$ & $\left(\frac{9}{4}, \frac{1}{4}\right)$ & $\frac{5}{4} \sqrt{2}$ \\
\hline 12 & {$[2,4,4,2]$} & $\frac{264}{12}$ & $\left(\frac{5}{2}, \frac{1}{2}\right)$ & $\frac{1}{2} \sqrt{10}$ & $\left(\frac{5}{2}, \frac{1}{2}\right)$ & $\frac{1}{2} \sqrt{10}$ \\
\hline 13 & {$[3,4,4,2]$} & $\frac{340}{13}$ & $\left(\frac{31}{13}, \frac{5}{13}\right)$ & $\frac{18}{13} \sqrt{2}$ & $\left(\frac{23}{10}, \frac{3}{10}\right)$ & $\frac{13}{10} \sqrt{2}$ \\
\hline 14 & {$[3,4,4,3]$} & $\frac{425}{14}$ & $\left(\frac{5}{2}, \frac{2}{7}\right)$ & $\frac{3}{14} \sqrt{85}$ & $\left(\frac{5}{2}, \frac{1}{6}\right)$ & $\frac{1}{6} \sqrt{130}$ \\
\hline 15 & {$[4,4,4,3]$} & $\frac{528}{15}$ & $\left(\frac{12}{5}, \frac{2}{5}\right)$ & $\frac{1}{5} \sqrt{113}$ & $\left(\frac{5}{2}, \frac{1}{2}\right)$ & $\frac{3}{2} \sqrt{2}$ \\
\hline $16 \mathrm{a}$ & {$[2,4,4,4,2]$} & $\frac{640}{16}$ & $\left(3, \frac{1}{2}\right)$ & $\frac{1}{2} \sqrt{17}$ & $\left(3, \frac{1}{2}\right)$ & $\frac{1}{2} \sqrt{17}$ \\
\hline $16 \mathrm{~b}$ & {$[4,4,4,4]$} & $\frac{640}{16}$ & $\left(\frac{5}{2}, \frac{1}{2}\right)$ & $\frac{3}{2} \sqrt{2}$ & $\left(\frac{5}{2}, \frac{1}{2}\right)$ & $\frac{3}{2} \sqrt{2}$ \\
\hline $17 \mathrm{a}$ & {$[4,5,5,3]$} & $\frac{780}{17}$ & $\left(\frac{41}{17}, \frac{2}{17}\right)$ & $\frac{40}{17}$ & $(2,0)$ & $\sqrt{5}$ \\
\hline $17 \mathrm{~b}$ & {$[2,4,5,4,2]$} & $\frac{780}{17}$ & $\left(3, \frac{6}{17}\right)$ & $\frac{40}{17}$ & $\left(3, \frac{1}{6}\right)$ & $\frac{13}{6}$ \\
\hline 18 & {$[3,4,5,4,2]$} & $\frac{925}{18}$ & $\left(\frac{26}{9}, \frac{5}{18}\right)$ & $\frac{1}{18} \sqrt{1685}$ & $(3,0)$ & $\sqrt{5}$ \\
\hline 19 & {$[3,5,5,4,2]$} & $\frac{1084}{19}$ & $\left(\frac{54}{19}, \frac{3}{19}\right)$ & $\frac{1}{19} \sqrt{1937}$ & $(3,0)$ & $\sqrt{5}$ \\
\hline 20 & {$[3,5,5,5,2]$} & $\frac{1255}{20}$ & $\left(\frac{29}{10}, \frac{1}{20}\right)$ & $\frac{1}{20} \sqrt{2165}$ & $(3,0)$ & $\sqrt{5}$ \\
\hline 21 & {$[3,5,5,5,3]$} & $\frac{1428}{21}$ & $(3,0)$ & $\sqrt{5}$ & $(3,0)$ & $\sqrt{5}$ \\
\hline 22 & {$[3,5,5,5,4]$} & $\frac{1664}{22}$ & $\left(\frac{34}{11}, \frac{1}{11}\right)$ & $\frac{21}{11} \sqrt{2}$ & $\left(\frac{45}{14}, \frac{3}{14}\right)$ & $\frac{25}{14} \sqrt{2}$ \\
\hline 23 & {$[5,5,5,5,3]$} & $\frac{1916}{23}$ & $\left(\frac{65}{23}, 0\right)$ & $\frac{2}{23} \sqrt{970}$ & $\left(\frac{21}{8}, 0\right)$ & $\frac{5}{8} \sqrt{17}$ \\
\hline 24 & {$[1,5,5,5,5,3]$} & $\frac{2183}{24}$ & $\left(\frac{89}{24}, 0\right)$ & $\frac{65}{24}$ & $\left(\frac{18}{5}, 0\right)$ & $\frac{13}{5}$ \\
\hline 25 & {$[3,5,5,5,5,2]$} & $\frac{2474}{25}$ & $\left(\frac{17}{5}, \frac{1}{25}\right)$ & $\frac{1}{25} \sqrt{4801}$ & $\left(\frac{7}{2}, 0\right)$ & $\frac{1}{2} \sqrt{29}$ \\
\hline 26 & {$[3,5,5,5,5,3]$} & $\frac{2769}{26}$ & $\left(\frac{7}{2}, 0\right)$ & $\frac{1}{2} \sqrt{29}$ & $\left(\frac{7}{2}, 0\right)$ & $\frac{1}{2} \sqrt{29}$ \\
\hline 27 & {$[1,4,6,6,6,4]$} & $\frac{3116}{27}$ & $\left(\frac{35}{9}, \frac{13}{27}\right)$ & $\frac{13}{27} \sqrt{37}$ & $\left(\frac{15}{4}, \frac{1}{2}\right)$ & $\frac{5}{4} \sqrt{5}$ \\
\hline 28 & {$[4,6,6,6,4,2]$} & $\frac{3464}{28}$ & $\left(\frac{45}{14}, \frac{1}{2}\right)$ & $\frac{1}{14} \sqrt{1570}$ & $\left(\frac{13}{4}, \frac{1}{2}\right)$ & $\frac{5}{4} \sqrt{5}$ \\
\hline 29 & {$[2,5,6,6,6,4]$} & $\frac{3852}{29}$ & $\left(\frac{108}{29}, \frac{12}{29}\right)$ & $\frac{10}{29} \sqrt{74}$ & $\left(\frac{7}{2}, \frac{1}{2}\right)$ & $\frac{1}{2} \sqrt{34}$ \\
\hline 30 & {$[4,6,6,6,5,3]$} & $\frac{4258}{30}$ & $\left(\frac{101}{30}, \frac{11}{30}\right)$ & $\frac{1}{30} \sqrt{7922}$ & $\left(\frac{7}{2}, \frac{1}{2}\right)$ & $\frac{1}{2} \sqrt{34}$ \\
\hline 31 & {$[3,6,6,6,6,4]$} & $\frac{4688}{31}$ & $\left(\frac{111}{31}, \frac{14}{31}\right)$ & $\frac{1}{31} \sqrt{8642}$ & $\left(\frac{7}{2}, \frac{1}{2}\right)$ & $\frac{1}{2} \sqrt{34}$ \\
\hline 32 & {$[4,6,6,6,6,4]$} & $\frac{5120}{32}$ & $\left(\frac{7}{2}, \frac{1}{2}\right)$ & $\frac{1}{2} \sqrt{34}$ & $\left(\frac{7}{2}, \frac{1}{2}\right)$ & $\frac{1}{2} \sqrt{34}$ \\
\hline $33 \mathrm{a}$ & {$[4,6,6,6,6,5]$} & $\frac{5680}{33}$ & $\left(\frac{118}{33}, \frac{14}{33}\right)$ & $\frac{80}{33} \sqrt{2}$ & $\left(\frac{67}{18}, \frac{5}{18}\right)$ & $\frac{41}{18} \sqrt{2}$ \\
\hline $33 \mathrm{~b}$ & {$[4,6,6,6,6,4,1]$} & $\frac{5680}{33}$ & $\left(\frac{119}{33}, \frac{16}{33}\right)$ & $\frac{80}{33} \sqrt{2}$ & $\left(\frac{23}{6}, \frac{1}{2}\right)$ & $\frac{1}{6} \sqrt{370}$ \\
\hline 34 & {$[4,6,7,7,6,4]$} & $\frac{6241}{34}$ & $\left(\frac{7}{2}, \frac{5}{17}\right)$ & $\frac{1}{34} \sqrt{12833}$ & $\left(\frac{7}{2}, \frac{1}{6}\right)$ & $\frac{1}{6} \sqrt{370}$ \\
\hline 35 & {$[5,6,7,7,6,4]$} & $\frac{6816}{35}$ & $\left(\frac{24}{7}, \frac{8}{35}\right)$ & $\frac{1}{35} \sqrt{13309}$ & $\left(\frac{129}{38}, \frac{7}{38}\right)$ & $\frac{1}{38} \sqrt{15170}$ \\
\hline 36 & {$[3,5,6,7,7,5,3]$} & $\frac{7406}{36}$ & $\left(\frac{145}{36}, \frac{1}{12}\right)$ & $\frac{1}{36} \sqrt{13546}$ & $(4,0)$ & $\sqrt{10}$ \\
\hline 37 & {$[3,5,7,7,7,5,3]$} & $\frac{7992}{37}$ & $(4,0)$ & $\sqrt{10}$ & $(4,0)$ & $\sqrt{10}$ \\
\hline 38 & {$[4,5,7,7,7,5,3]$} & $\frac{8689}{38}$ & $\left(\frac{149}{38}, \frac{1}{19}\right)$ & $\frac{37}{38} \sqrt{13}$ & $\left(\frac{23}{6}, \frac{1}{6}\right)$ & $\frac{1}{6} \sqrt{410}$ \\
\hline 39 & {$[3,5,7,7,7,6,4]$} & $\frac{9388}{39}$ & $\left(\frac{161}{39}, \frac{5}{39}\right)$ & $\frac{1}{39} \sqrt{17873}$ & $\left(\frac{25}{6}, \frac{1}{6}\right)$ & $\frac{1}{6} \sqrt{410}$ \\
\hline 40 & {$[3,6,7,7,7,6,4]$} & $\frac{10127}{40}$ & $\left(\frac{163}{40}, \frac{1}{5}\right)$ & $\frac{1}{40} \sqrt{19433}$ & $\left(\frac{235}{58}, \frac{15}{58}\right)$ & $8 \sqrt{3944}$ \\
\hline
\end{tabular}

